\title{
FREE CONVECTIVE MAGNETO-NANOFLUID FLOW PAST A MOVING VERTICAL PLATE IN THE PRESENCE OF RADIATION AND THERMAL DIFFUSION
}

\author{
P. Chandra Reddy ${ }^{1}$, M.C. Raju ${ }^{1,}{ }^{*}$, G.S.S. Raju ${ }^{2}$, S.V.K. Varma ${ }^{3}$ \\ ${ }^{1}$ Department of Mathematics, Annamacharya Institute of Technology and Sciences (Autonomous), Rajampet-516126, A.P., India. \\ ${ }^{2}$ Department of Mathematics, JNTUA College of Engineering, Pulivendula, A.P., India. \\ ${ }^{3}$ Department of Mathematics, S. V. University, Tirupati -517502, A.P., India.
}

\begin{abstract}
The present analysis is focused on free convective heat and mass transfer characteristics of magneto-nanofluid flow through a moving vertical plate in the presence of thermal radiation and thermal diffusion. The water-based nanofluid containing copper is taken into consideration. A uniform magnetic field is applied perpendicular to the plate. The governing equations are solved by applying finite difference method. Numerical results of the fluid velocity, temperature, concentration, shear stress, rate of heat transfer and rate of mass transfer are presented graphically for different values of the physical parameters encountered in the problem. It is noticed that the fluid velocity increases with increasing values of radiation parameter and Soret number. An increase in radiation parameter and solid volume fraction leads to enhance the fluid temperature. Rising values of Soret number serves to improve the species concentration. Increasing values of Schmidt number enhanced the shear stress at the plate but a reverse trend is found in the case of Soret number. The current study is well supported by the verification of a previous result.
\end{abstract}

Keywords: Free convection, Magneto-nanofluid, Moving vertical plate, Thermal radiation and Soret effect.

\section{INTRODUCTION}

Nanofluids have many applications in the industries because of their unique physical and chemical properties. Choi (1995) first introduced the term nanofluid. Nanofluids are solid-liquid composite materials consisting of solid nanoparticles with sizes typically of 1$100 \mathrm{~nm}$ suspended in liquid. In the recent years, nanofluids have attracted many researchers due to the significance in their thermal properties. The traditional fluids like water, ethylene glycol and mineral oils have a low thermal conductivity where nanofluids have relatively higher thermal conductivity. Eastman et al. (1997) studied experimentally and reported that an increase in thermal conductivity of approximately $60 \%$ can be gained for a nanofluid consisting of water and 5\% volume of Copper nanoparticles. This phenomenon attributed to the increase in surface area due to the suspension of nanoparticles in the water. Later it was examined that a small amount (below 1\% volume fraction) of copper nanoparticles or carbon nanotube dispersed in ethylene glycol or oil can increase their inherently poor thermal conductivity by $40 \%$ and $50 \%$ respectively (2001). It was found that copper $(\mathrm{Cu})$ has a thermal conductivity 700 times more than water and 3000 times higher than engine oil. Das et al. (2003) established a two-to fourfold increase in thermal conductivity for water-based nanofluids containing $\mathrm{Al}_{2} \mathrm{O}_{3}$ or $\mathrm{CuO}$ nanoparticles over a small temperature range $21-51^{\circ} \mathrm{C}$. Masuda et al. (1993) shown that a characteristic feature of the nanoparticle is to increase the thermal conductivity of the fluid. The concept of heat transfer in nanofluids has been surveyed and reported in the articles by Das and Choi (2009), Kakac and Pramuanjaroenkij (2009), Sheikholeslami et al. (2014,2015), Kandelousi (2014), Sheikholeslami and Ganji (2014), Sheikholeslami and Gorji-Bandpya (2014).

Magnetohydrodynamic (MHD) flow with heat and mass transfer has essential applications for real world problems in physics, chemistry and engineering. Industrial apparatus (machines) such as magnetohydrodynamic (MHD) pumps, generators, motors, hydraulic machines, bearings and boundary layer control are very much affected by the interaction between the electrical conduction of the fluid and magnetic field effects. The most important problem in this area is the hydromagnetic behavior of boundary layers along fixed or moving surfaces in the presence of a transverse magnetic field. MHD boundary layers are considered and they implemented effectively in various technical fields employing liquid metals as well as plasma flows in magnetic fields. In the recent years, many researchers contributed in studying the influences of electrically conducting nanofluids in the presence of a magnetic field on the flow and heat transfer of an incompressible viscous fluid. Nanofluids such as water mixed with little acid and other ingredients are considered. Hamad and Pop (2011) studied unsteady MHD natural convection flow past a vertical permeable flat plate in a rotating frame of reference in a nanofluid with constant heat source. Also Hamad et al. (2010) examined the magnetic field effects on free convection flow of a nanofluid through a semi-infinite vertical flat plate. Hayat et al. (2010) discussed thermal diffusion and Diffusion thermo effects on 


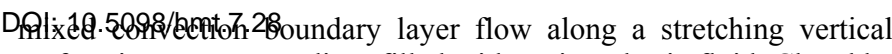
surface in a porous medium filled with a viscoelastic fluid. Chamkha and Aly (2011) analyzed MHD free convection flow of a nanofluid past a vertical plate with the influence of heat generation or absorption effects. Turkyilmazoglu (2012) presented exact analytical solutions for heat and mass transfer effects on magnetohydrodynamic slip flow in nanofluids. Vajravelu et al. (2013) considered and analyzed an unsteady boundary layer flow of a convective viscous fluid over a vertical surface with changeable fluid properties. Prakash et al. (2013) studied theoretically radiation and diffusion-thermo effects on unsteady MHD flow of an impulsively started infinite vertical porous plate with changeable temperature and mass diffusion. Seth et al. (2010) discussed Natural convection MHD fluid flow past an impetuously moving vertical plate with the implementation of ramped wall temperature under the influence of thermal diffusion and heat absorption. Khan et al. (2014) analyzed the properties of MHD boundary layer nanofluid flow consisting of gyro tactic microbes past a vertical plate with the consideration of Navier slip. Srinivasacharya and Kaladhar (2012a) analyzed Hall and Ion-Slip effects on mixed convection flow of couple stress fluid between parallel vertical plates. Later Kaladhar and Srinivasacharya (2014) established the effects of thermal and also solutal stratification on free and forced convection flow over a vertical plate flooded with couple stress fluid. Srinivas and Ramana Murthy (2015) analyzed and discussed thermodynamic MHD flow of two immiscible micropolar fluid between two parallel plates. Mahian et al. (2012) examined and analyzed first and second laws of thermodynamics in the presence of MHD flow linking two isothermal cylinders with relative rotation. $\mathrm{Yu}$ et al. (2012) examined the effect of radiation on natural convective radial heat absorption. Umamaheswar et al. (2015) studied MHD transitory free convection flow of a Newtonian fluid past an infinite vertical porous plate. Ferdows et al. (2012) considered and analyzed MHD mixed convective boundary layer flow of a nanofluid through a porous medium due to an exponentially stretching sheet. Ferdows et al. (2013) performed a numerical study of transient MHD radiative free convection nanofluid flow from a stretching permeable surface. Khan et al. (2014) discussed MHD boundary layer radiative, heat generating and chemical reacting flow past a wedge moving in a nanofluid. Further, they examined heat generation effects on unsteady mixed convection flow from a vertical porous plate with induced magnetic field. Wahiduzzaman et al. (2015) analyzed viscous dissipation and radiation effects on MHD boundary layer flow of a nanofluid past a rotating stretching sheet. In addition, they established MHD convective stagnation flow of nanofluid over a shrinking surface with thermal radiation, heat generation and chemical reaction. Khan et al. (2012) established MHD free convection boundary layer unsteady flow of a nanofluid along a stretching sheet with thermal radiation and viscous dissipation effects. Beg et al. (2014) examined and reported explicit numerical study of unsteady hydromagnetic mixed convective nanofluid flow from an exponential stretching sheet in porous media.

Keeping in view the above studies, we attempted to study the characteristics of magneto hydrodynamic free convective nanofluid flow past a moving vertical plate in the presence of radiation and thermal diffusion. We have extended the work of Das and Jana (2015) with the novelty of considering the mass diffusion and thermal diffusion. The water-based nanofluid containing nanoparticles of copper $(\mathrm{Cu})$ have been considered in the current study. The governing equations along with the boundary conditions have solved by finite difference method and the effects of various parameters have analyzed through graphical representations.

\section{FORMULATION OF THE PROBLEM}

We have considered an unsteady free convective flow, heat and mass transfer of a magneto-nanofluid past an infinite vertical flat plate that moves with an impulsive motion. At time $\mathrm{t}^{*}=0$, the plate is assumed to be at rest with the constant ambient temperature $T_{\infty}^{*}$ and the concentration $C_{\infty}^{*}$. At time $\mathrm{t}^{*}>0$, the plate start ISN: 2151 - 8629 own plane with the velocity $\lambda u_{0}$ in the vertical direction, where $u_{0}$ is constant and the temperature of the plate is raised or lowered to $T_{w}^{*}$ and the level of concentration is also maintained at $C_{w}^{*}$. We choose the $\mathrm{x}$-axis along the plate in the upward direction vertically and $\mathrm{y}$ axis is taken perpendicular to it. A uniform transverse magnetic field of strength $\mathrm{B}_{0}$ is applied perpendicular to the plate (parallel to the $\mathrm{y}-$ axis). The plate coincides with the plane $y=0$ and the fluid flow being restricted to $y>0$. It has assumed that the pressure gradient has neglected in this analysis. It is also assumed that a radiative heat flux $\mathrm{q}_{\mathrm{r}}$ is applied in the normal direction to the plate and mass flux caused by the temperature differences which is termed as thermal diffusion effect is considered. The fluid is water-based nanofluid containing nanoparticles of Copper $(\mathrm{Cu})$. It has also assumed that the base fluid and the suspended nanoparticles are in thermal equilibrium. The values related to thermo physical properties of the cu-water nanofluid are presented in Table 1 . We assume that the density is linearly dependent on temperature buoyancy forces in the equations of flow pattern. This consideration is exact enough for both dropping liquid and gases for minor values of the temperature difference.

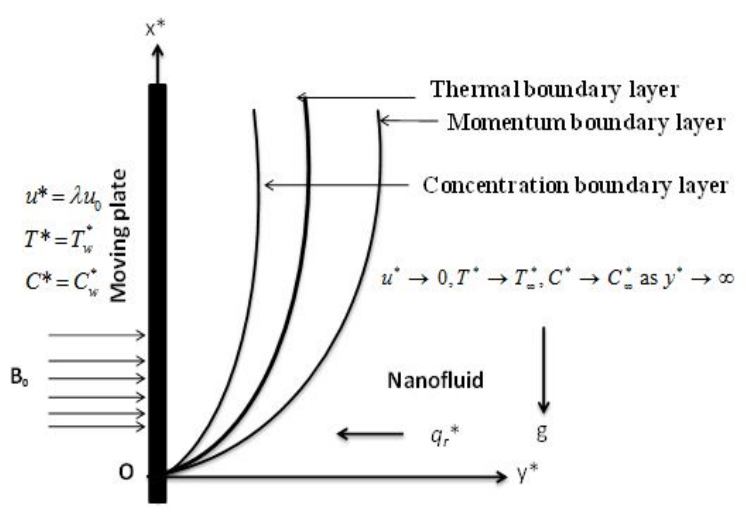

Fig. 1 Physical model and coordinate system

It has assumed that induced magnetic field generated by the fluid flow is negligible in comparison with the applied one so that we assume magnetic field $\vec{B} \equiv\left(0,0, B_{0}\right)$. This assumption is relevant since the magnetic Reynolds number is very less for metallic liquids and partially ionized fluids. Further, no external electric field has applied such that the effect of polarization of fluid is negligible (2015) and hence we assume $\vec{E}=(0,0,0)$. Under the above assumptions, the momentum, energy and concentration equations in the presence of magnetic field, thermal radiation and thermal diffusion past a moving vertical plate are expressed as

$$
\begin{gathered}
\rho_{n f} \frac{\partial u^{*}}{\partial t^{*}}=\mu_{n f} \frac{\partial^{2} u^{*}}{\partial y^{* 2}}+g(\rho \beta)_{n f}\left(T^{*}\right. \\
g\left(\rho \beta^{*}\right)_{n f}\left(C^{*}-C_{\infty}^{*}\right) \\
\left(\rho C_{p}\right)_{n f} \frac{\partial T^{*}}{\partial t^{*}}=k_{n f} \frac{\partial^{2} T^{*}}{\partial y^{*^{2}}}-\frac{\partial q_{r}^{*}}{\partial y} \\
\frac{\partial C^{*}}{\partial t^{*}}=D \frac{\partial^{2} C^{*}}{\partial y^{* 2}}+D_{1} \frac{\partial^{2} T^{*}}{\partial y^{* 2}}
\end{gathered}
$$


temperature. This is done by expanding $T^{4}$ in a TayldSSN free stream temperature $T_{\infty}$ as follows:

where

$$
\begin{aligned}
& \mu_{n f}=\frac{\mu_{f}}{(1-\phi)^{2.5}}, \quad \rho_{n f}=(1-\phi) \rho_{f}+\phi \rho_{S}, \\
& \left(\rho C_{p}\right)_{n f}=(1-\phi)\left(\rho C_{p}\right)_{f}+\phi\left(\rho C_{p}\right)_{S}, \\
& (\rho \beta)_{n f}=(1-\phi)(\rho \beta)_{f}+\phi(\rho \beta)_{S}, \\
& \left(\rho \beta^{*}\right)_{n f}=(1-\phi)\left(\rho \beta^{*}\right)_{f}+\phi\left(\rho \beta^{*}\right)_{S}, \\
& \sigma_{n f}=\sigma_{f}\left[1+\frac{3(\sigma-1) \phi}{(\sigma+2)-(\sigma-1) \phi}\right], \sigma=\frac{\sigma_{S}}{\sigma_{f}},
\end{aligned}
$$

It is worth mentioning that the expressions (1) are restricted to spherical nanoparticles, where it does not account for other shapes of nanoparticles. The effective thermal conductivity of the nanofluid given by Hamilton and Crosser model followed by Kakac and Pramuanjaroenkij [8] is given by

$k_{n f}=k_{f}\left[\frac{k_{S}+2 k_{f}-2 \phi\left(k_{f}-k_{S}\right)}{k_{S}+2 k_{f}+\phi\left(k_{f}-k_{S}\right)}\right]$

where $k_{f}$ is the thermal conductivity of the base fluid and $k_{s}$ the thermal conductivity of the nanoparticle. In Eqs. (1) - (5), the subscripts $n f, f$ and $s$ denote the thermo physical properties of the nanofluid, base fluid and nanoparticles, respectively.

The initial and boundary conditions are

$$
\begin{aligned}
& t^{*}=0: u^{*}=0, T^{*}=T_{\infty}^{*}, C^{*}=C_{\infty}^{*} \text { for all } y^{*} \geq 0, \\
& t^{*}>0: u^{*}=\lambda u_{0}, T^{*}=T_{w}^{*}, C^{*}=C_{w}^{*} \text { at } y^{*}=0, \\
& t^{*}>0: u^{*} \rightarrow 0, T^{*} \rightarrow T_{\infty}^{*}, C^{*} \rightarrow C_{\infty}^{*} \text { as } y^{*} \rightarrow \infty
\end{aligned}
$$

where $\lambda$ indicates the direction of the moving plate with $\lambda=0$ for the stationary plate, while $\lambda= \pm 1$ for the forth and back movement of the plate.

Table 1: Thermo physical properties of water and nanoparticle (2015).

\begin{tabular}{|l|c|c|}
\hline Physical properties & Water/base fluid & Cu(copper) \\
\hline$\rho\left(\mathrm{kg} / \mathrm{m}^{3}\right)$ & 997.1 & 8933 \\
\hline$C_{p}(\mathrm{~J} / \mathrm{kg} \mathrm{K})$ & 4179 & 385 \\
\hline $\mathrm{k}(\mathrm{W} / \mathrm{m} \mathrm{K})$ & 0.613 & 401 \\
\hline$\beta \times 10^{5}\left(\mathrm{~K}^{-1}\right)$ & 21 & 1.67 \\
\hline$\phi$ & 0.0 & 0.05 \\
\hline$\sigma(S / \mathrm{m})$ & $5.5 \times 10^{-6}$ & $59.6 \times 10^{6}$ \\
\hline
\end{tabular}

It is assumed that the fluid is an optically thick (photon mean free path is very small) gray gas (which emits and absorbs but does not scatter thermal radiation). In an optically thick medium, the radiation penetration length is small compare to the characteristic length. The photon mean path gives the average distance travelled by a moving photon between successive collisions, which modify its direction or energy or other particle properties. For an optically thick fluid, we can adopt Rosseland approximation for radiative flux. The Rosseland approximation from Das and Jana (2015) applies to optically thick media and gives the net radiation heat flux by the expression

$$
q_{r}^{*}=-\frac{4 \sigma^{*}}{3 k^{*}} \frac{\partial T^{4}}{\partial y}
$$

where $\sigma^{*}\left(=5.67 \times 10^{-8} W / m^{2} K^{4}\right) \quad$ is the Stefan-Boltzmann constant and $k^{*}\left(\mathrm{~m}^{-1}\right)$ the Rosseland mean absorption coefficient. We assume that the temperature difference within the flow is sufficiently small such that the term $T^{4}$ may be expressed as a linear function of
$T^{4}=T_{\infty}^{4}+3 T_{\infty}^{3}\left(T-T_{\infty}\right)+6 T_{\infty}^{2}\left(T-T_{\infty}\right)^{2}+\ldots \ldots$

Neglecting higher-order terms in equation (8) beyond the first order in $\left(T-T_{\infty}\right)$, we get

$T^{4} \approx 4 T_{\infty}^{3} T-3 T_{\infty}^{4}$

Using the Eq.s (7) and (9), Eq. (2) becomes

$\frac{\partial T^{*}}{\partial t^{*}}=\frac{1}{\left(\rho C_{p}\right)_{n f}}\left(k_{n f}+\frac{16 \sigma^{*} T_{\infty}^{3}}{3 k^{*}}\right) \frac{\partial^{2} T^{*}}{\partial y^{* 2}}$

By introducing the non-dimensional variables

$y=\frac{u_{0} y^{*}}{v_{f}}, t=\frac{u_{0}^{2} t^{*}}{v_{f}}, u=\frac{u^{*}}{u_{0}}, \theta=\frac{T^{*}-T_{\infty}^{*}}{T_{w}^{*}-T_{\infty}^{*}}, C=\frac{C^{*}-C_{\infty}^{*}}{C_{w}^{*}-C_{\infty}^{*}}$

Eq.s (1), (3) and (10) reduces to the form

$\frac{\partial u}{\partial t}=a_{1} \frac{\partial^{2} u}{\partial y^{2}}+G r a_{2} \theta+G c a_{5} C-M^{2} a_{3} u$

$\frac{\partial \theta}{\partial t}=a_{4} \frac{\partial^{2} \theta}{\partial y^{2}}$

$\frac{\partial C}{\partial t}=\frac{1}{S C} \frac{\partial^{2} C}{\partial y^{2}}+S o \frac{\partial^{2} \theta}{\partial y^{2}}$

where

$$
\begin{aligned}
& x_{1}=\left[(1-\phi)+\phi\left(\frac{\rho_{S}}{\rho_{f}}\right)\right], x_{2}=\left[(1-\phi)+\phi\left(\frac{(\rho \beta)_{S}}{(\rho \beta)_{f}}\right)\right], \\
& x_{3}=\left[(1-\phi)+\phi\left(\frac{\left(\rho C_{p}\right)_{S}}{\left(\rho C_{p}\right)_{f}}\right)\right], x_{4}=\left[\frac{k_{S}+2 k_{f}-2 \phi\left(k_{f}-k_{S}\right)}{k_{S}+2 k_{f}+\phi\left(k_{f}-k_{S}\right)}\right], \\
& x_{5}=\left[1+\frac{3(\sigma-1) \phi}{(\sigma+2)-(\sigma-1) \phi}\right], x_{6}=\frac{x_{4}}{x_{3}}, \\
& x_{7}=\left[(1-\phi)+\phi\left(\frac{\left(\rho \beta^{*}\right)_{S}}{\left(\rho \beta^{*}\right)}\right)\right], a_{1}=\frac{1}{(1-\phi)^{2.5} x_{1}}, \\
& a_{2}=\frac{x_{2}}{x_{1}}, a_{3}=\frac{x_{5}}{x_{1}}, a_{4}=\frac{1}{x_{3} \operatorname{Pr}}\left(x_{4}+N r\right), a_{5}=\frac{x_{7}}{x_{1}}
\end{aligned}
$$

$M^{2}=\frac{\sigma_{f} B_{0}^{2} v_{f}}{\rho_{f} u_{0}^{2}} \quad$ (Magnetic parameter),

$R=\frac{16 \sigma^{*} T_{\infty}^{3}}{3 k_{f} k^{*}} \quad$ (Radiation parameter),

$\operatorname{Pr}=\frac{\mu_{f}{ }^{C} p}{{ }_{f}} \quad$ (Prandtl number),

$G r=\frac{g \beta_{f} \mu_{f}\left(T_{w}^{*}-T_{\infty}^{*}\right)}{u_{0}^{3}}$ (Grashof number),

$G c=\frac{g\left(\beta^{*}\right) f^{\mu} f^{\left(C_{w}^{*}-C_{\infty}^{*}\right)}}{u_{0}^{3}}$ (Modified Grashof number),

Sc $=\frac{v_{f}}{D} \quad$ (Schmidt number),

$S o=\frac{D_{1}}{v_{f}}\left(\frac{T_{w}^{*}-T_{\infty}^{*}}{C_{w}^{*}-C_{\infty}^{*}}\right) \quad$ (Soret number).

The corresponding initial and boundary conditions are

$t=0: u=0, C=0, \theta=0$ for all $y \geq 0$,

$t>0: u=\lambda, C=1, \theta=1, \quad$ at $y=0$, 
Equations (12)-(14) are linear partial differential equations and are to be solved with the initial and boundary conditions (15). For this set of equations the exact solution is not possible and hence we solve these equations by finite-difference method. For this, a rectangular region of the flow field is chosen, and the region is divided into a grid of lines parallel to $\mathrm{X}$ and $\mathrm{Y}$-axes, where the $\mathrm{X}$-axis is taken along the plate and the $\mathrm{Y}$-axis is normal to the plate as shown in Fig.2.

The equivalent finite difference schemes of equations for (12)-(14) are as follows:

$$
\begin{aligned}
& \begin{array}{l}
\frac{u_{i, j+1}-u_{i, j}}{\Delta t}=a_{1} \frac{u_{i-1, j}-2 u_{i, j}+u_{i+1, j}}{(\Delta y)^{2}}+G r a_{2} \theta_{i, j} \\
+G c a_{5} C_{i, j}-M^{2} u_{i, j}
\end{array} \\
& \begin{array}{c}
\frac{\theta_{i, j+1}-\theta_{i, j}}{\Delta t}=a_{4} \frac{\theta_{i-1, j}-2 \theta_{i, j}+\theta_{i+1, j}}{(\Delta y)^{2}} \\
\frac{C_{i, j+1}-C_{i, j}}{\Delta t}=\frac{1}{S c} \frac{C_{i-1, j}-2 C_{i, j}+C_{i+1, j}}{(\Delta y)^{2}}+S o \frac{\theta_{i-1, j}-2 \theta_{i, j}+\theta_{i+1, j}}{(\Delta y)^{2}}
\end{array}
\end{aligned}
$$

Here, the suffix i refer to $y$ and $\mathrm{j}$ to time. The mesh system is divided by taking $\Delta y=0.1$. From the initial condition in (15), we have the following equivalent:

$$
u(i, 0)=0, \theta(i, 0)=0, C(i, 0)=0 \text { for all } i
$$

The boundary conditions from (15) are expressed in finite-difference form as follows

$u(0, j)=\lambda, \theta(0, j)=1, C(0, j)=1$ for all $j$

$u\left(i_{\max }, j\right)=0, \theta\left(i_{\max }, j\right)=0, C\left(i_{\max }, j\right)=0$ for all $j$

(Here $i_{\max }$ was taken as 200)

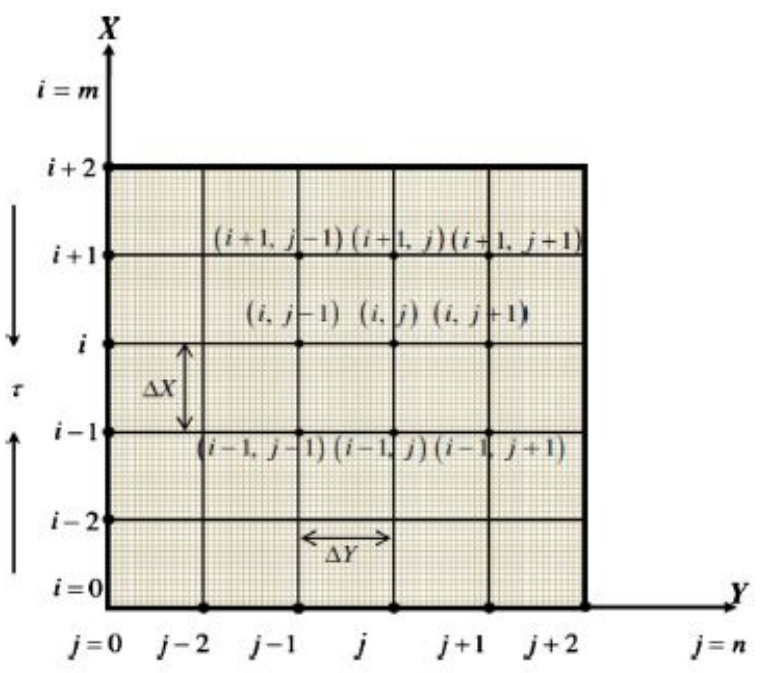

Fig. 2 Finite difference space grid

The velocity at the end of time step viz, $u(i, j+1)(i=1,200)$ is computed from (9) in terms of velocity, temperature and concentration at points on the earlier time-step. After that $\theta(i, j+1)$ is computed from (10) and then $C(i, j+1)$ is computed from (11). The procedure is repeated until $\mathrm{t}=0.5$ (i.e. $\mathrm{j}=500$ ). During computation $\Delta \mathrm{t}$ was chosen as 0.001 .

\section{RESULTS AND DISCUSSION}

In order to gain a perspective of the physics of the flow regime, a parametric study has performed and the numerical results have elucidated with the help of graphs. We have analyzed the nondimensional fluid velocity $\mathrm{u}$, the fluid temperature $\theta$ and the concentration of the fluid $\mathrm{C}$ for several values of magnetic parameter M, Grashof number Gr, modified Grashof number Gc, radiation

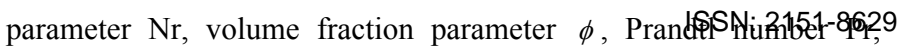
Schmidt number Sc and Soret number So in figures 3-16. To confirm the accuracy of the methodology, our present study has compared with the study of Das and Jana (2015) by considering the effect of radiation parameter on temperature distribution. The results of this comparison are found to be in very good agreement. It is evident from Fig. 3.

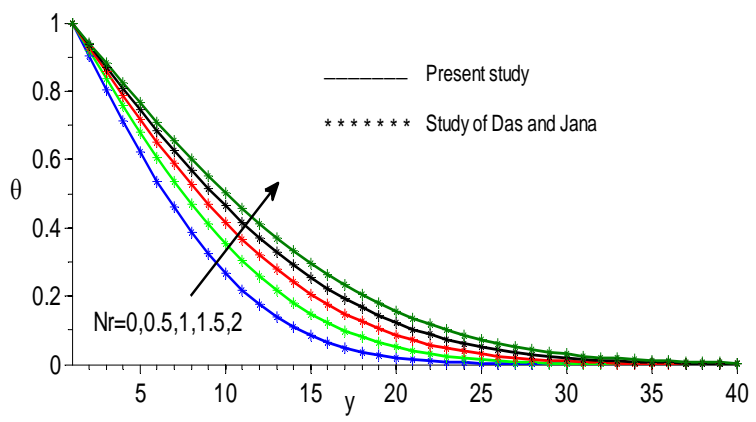

Fig. 3 Comparison of the results: Effect of radiation parameter on temperature distribution in the absence of Gc, Sc and So.

\subsection{Effects of the parameters on velocity profiles:}

Figure 4 presents the effect of Grashof number on velocity distribution. It is noticed that the velocity $u$ increases with increasing values of Grashof number $\mathrm{Gr}$ for the cases of stationary plate $(\lambda=0)$ as well as moving plate $(\lambda= \pm 1)$. This behavior is because the positive Grashof number $\mathrm{Gr}$ acts like a favorable pressure gradient that accelerates the fluid in the boundary layer. As a result the velocity increases with Gr. Grashof number represents the effect of free convection currents. $\mathrm{Gr}=0$ corresponds the absence of free convection current. Fig. 5 displays the effect of Grashof number of mass transfer on velocity. It is observed that the velocity increases for increasing values of $\mathrm{Gc}$ for both the cases of stationary plate $(\lambda=0)$ and moving plate $(\lambda= \pm 1)$. This occurs because of the presence of thermal and solutal buoyancy, which has the tendency of increase in velocity. The effect of radiation parameter $\mathrm{Nr}$ on the velocity has demonstrated in Fig. 6. The velocity of the fluid enhances as the value of $\mathrm{Nr}$ increases for both the cases of stationary plate $(\lambda=0)$ and moving plate $(\lambda= \pm 1)$. The velocity distribution increase sharply near the surface of the plate and after attaining respective maximals, the curves come down to the corresponding asymptotic value. Therefore, $\mathrm{Nr}$ behaves like a supporting force that accelerates the fluid particles near the vicinity of the plate. It is observed from Fig. 7 that the velocity decreases with increasing values of Prandtl number for both the cases of stationary plate $(\lambda=0)$ and moving plate $(\lambda= \pm 1)$. This is because the fluid of low Prandtl number has high thermal diffusivity and hence attains higher temperature in steady state, which in turn means more buoyancy force. Fig. 8 reveals that the fluid velocity accelerates for increasing values of magnetic parameter. The momentum boundary layer thickness decreases for increasing values of $\mathrm{M}$ for the cases of stationary plate $(\lambda=0)$ as well as moving plate $(\lambda= \pm 1)$. This trend is due to fact that the transverse magnetic field sets in Lorentz force, which The Schmidt number embodies the ratio of the momentum diffusivity to the species (mass) diffusivity. It physically relates to the comparative thickness of the hydrodynamic boundary layer and mass-transfer boundary layer. It is observed that the velocity field decreases when retards the fluid velocity. Fig. 9 displays the effect of Schmidt number on fluid velocity. Schmidt number increases for the cases of stationary plate $(\lambda=0)$ as well as moving plate $(\lambda= \pm 1)$. The influence of Soret number on velocity field has demonstrated in Fig. 10. It is clear that the velocity enhanceswith the rising values of Soret number for the cases of stationary plate $(\lambda=0)$ as well as moving plate $(\lambda= \pm 1)$. Fig. 11 reveals that as time elapses, the velocity of the fluid increases for the cases of stationary plate $(\lambda=0)$ as well as moving plate $(\lambda= \pm 1)$. 


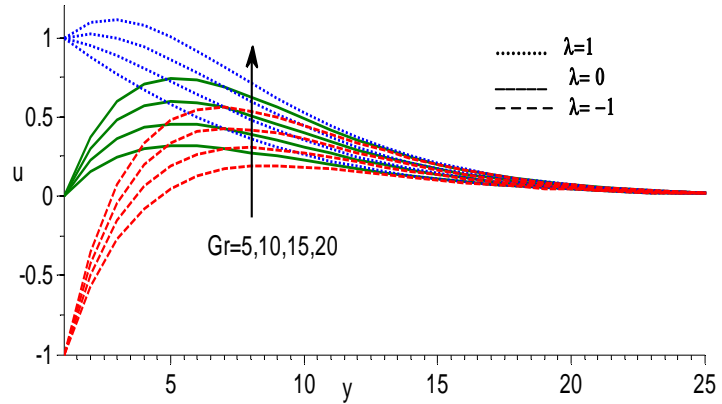

Fig. 4 Effect of Grashof number on velocity for $\mathrm{Gc}=5, \mathrm{Nr}=0.5, \mathrm{M}^{2}=10, \mathrm{Pr}=0.71, \mathrm{Sc}=0.22, \mathrm{~S}_{0}=1$.

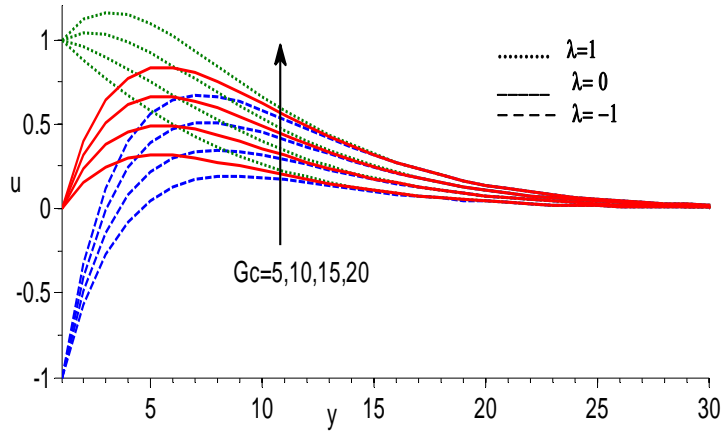

Fig. 5 Effect of modified Grashof number on velocity for $\mathrm{Gr}=5, \mathrm{Nr}=0.5, \mathrm{M}^{2}=10, \mathrm{Pr}=0.71, \mathrm{Sc}=0.22, \mathrm{So}=1$.

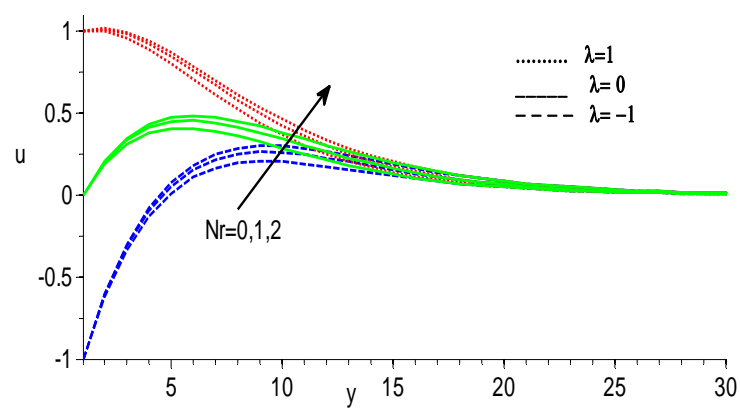

Fig. 6 Effect of radiation parameter on velocity for $\mathrm{Gr}=5, \mathrm{Gc}=5, \mathrm{M}^{2}=10, \mathrm{Pr}=0.71, \mathrm{Sc}=0.22, \mathrm{So}=1$.

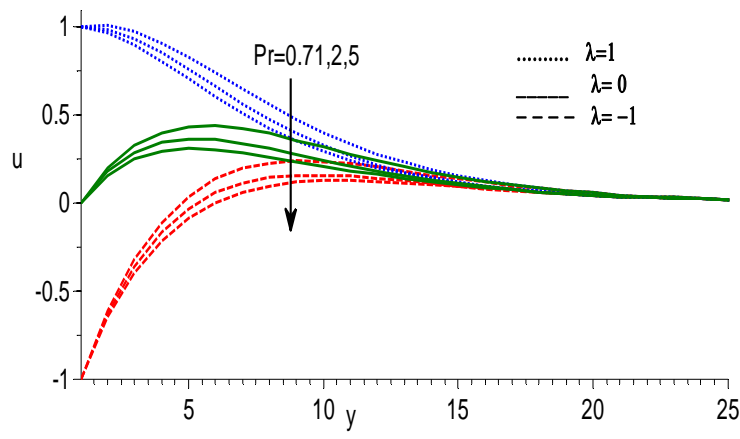

Fig. 7 Effect of Prandtl number on velocity for $\mathrm{Gr}=5, \mathrm{Gc}=5, \mathrm{Nr}=0.5$, $\mathrm{M}^{2}=10, \mathrm{Sc}=0.22, \mathrm{~S}_{0}=1$.

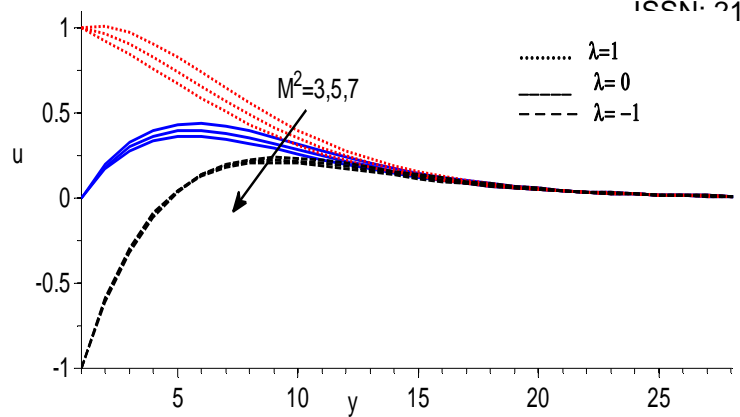

Fig. 8 Effect of magnetic parameter on velocity for $\mathrm{Gr}=5, \mathrm{Gc}=5, \mathrm{Pr}=0.71, \mathrm{Nr}=0.5, \mathrm{Sc}=0.22, \mathrm{So}=1$.

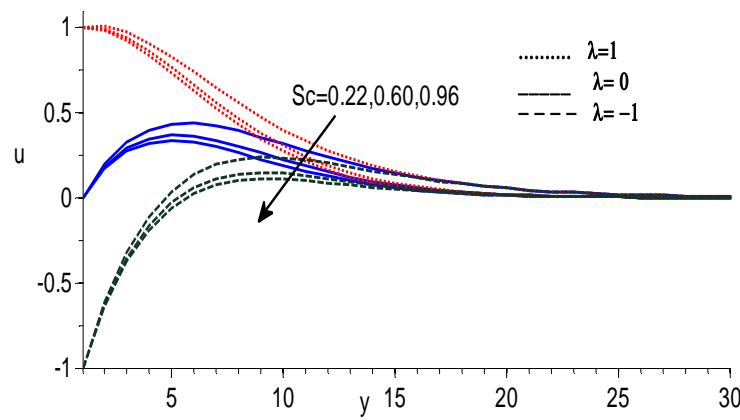

Fig. 9 Effect of Schmidt number on velocity for $\mathrm{Gr}=5$, $\mathrm{Gc}=5, \mathrm{M}^{2}=10, \mathrm{Pr}=0.71, \mathrm{Nr}=0.5, \mathrm{~S}_{0}=1$.



Fig. 10 Effect of Soret number on velocity for $\mathrm{Gr}=5$, $\mathrm{Gc}=5, \mathrm{Pr}=0.71, \mathrm{Nr}=0.5, \mathrm{M}^{2}=10, \mathrm{Sc}=0.22$.

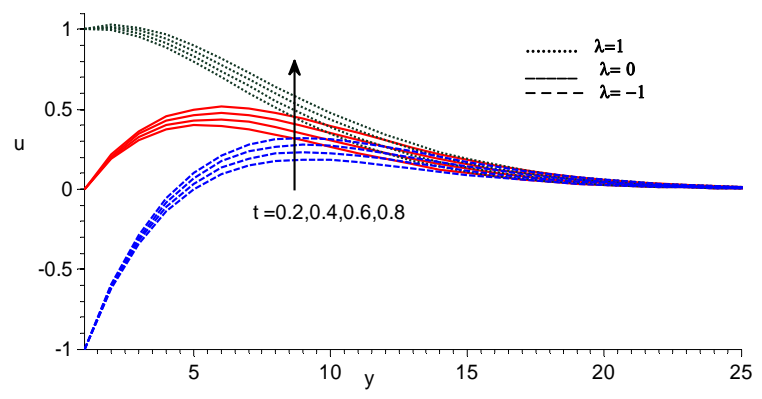

Fig. 11 Effect of time on velocity for $\mathrm{Gr}=5$, $\mathrm{Gc}=5, \mathrm{Pr}=0.71, \mathrm{Nr}=0.5, \mathrm{M}^{2}=10, \mathrm{Sc}=0.22$ 


\subsection{Effects of the parameters on temperature profiles:}

Figure 12 illustrates the effect of radiation parameter $\mathrm{Nr}$ on the temperature distribution. The fluid temperature increases as the value of $\mathrm{Nr}$ enlarges. The increase in radiation parameter corresponds to the release of heat energy from the flow region. A decrease in the values of $\mathrm{Nr}$ for given $\mathrm{knf}_{\mathrm{nf}}$ and $\mathrm{T}_{\infty}$ means a decrease in the Rosseland radiation absorptive $\mathrm{k}^{*}$. Since divergence of the radiative heat flux increases, $\mathrm{k}^{*}$ decreases which in turn causes to increase the rate of radiative heat transfer of the fluid and so the fluid temperature increases. This result is in good agreement with the results of Reddy et al. (2016). Fig. 13 represents the influence of solid volume fraction on temperature variations. It is clear that the fluid temperature rises with enlarging values of volume fraction parameter. The thermal boundary layer for $\mathrm{Cu}$-water nanofluid is higher than for pure water $(\phi=0)$ since copper has more thermal conductivity and its addition to the water based fluid increases the thermal conductivity for the fluid. Hence, the thickness of the thermal boundary layer enhances. This trend coincides with the physical nature of nanoparticles. This observation shows the significance of nanofluids in the cooling and heating processes. Fig. 14 represents the influence of time on temperature variations. It is clear that the fluid temperature rises with enlarging values of time. The effect of Prandtl number on the variation of fluid temperature has exhibited in Fig. 15. It has noticed that the fluid temperature falls down as the Prandtl number enlarges. It is obvious that a higher Prandtl number fluid has relatively low thermal conductivity, which causes a reduction in the conduction and there by the thermal boundary layer thickness. As a result, the fluid temperature decreases.

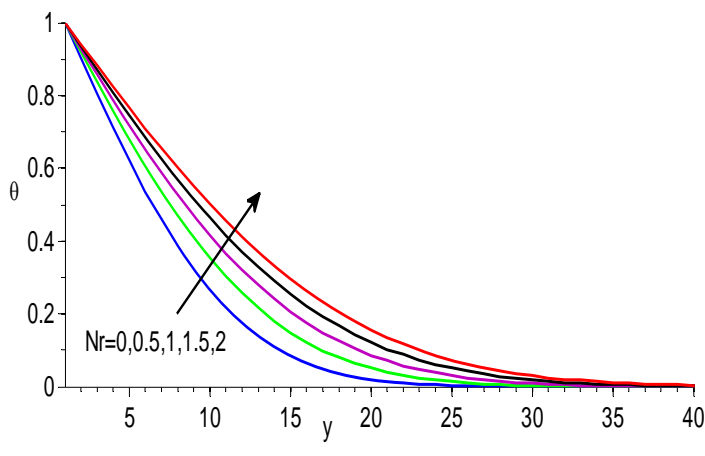

Fig. 12 Effect of radiation parameter on temperature for $\mathrm{Gr}=5, \mathrm{Gc}=5, \mathrm{M}^{2}=10, \operatorname{Pr}=0.71$.

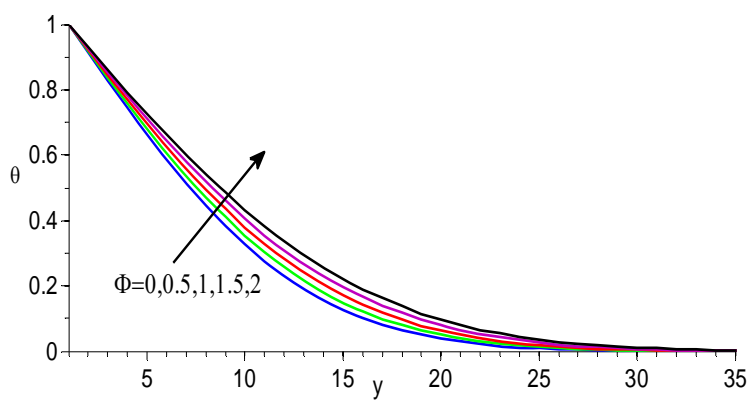

Fig. 13 Effect of solid volume fraction on temperature for $\mathrm{Gr}=5, \mathrm{Gc}=5, \mathrm{M}^{2}=10, \mathrm{Pr}=0.71, \mathrm{Nr}=0.5$.

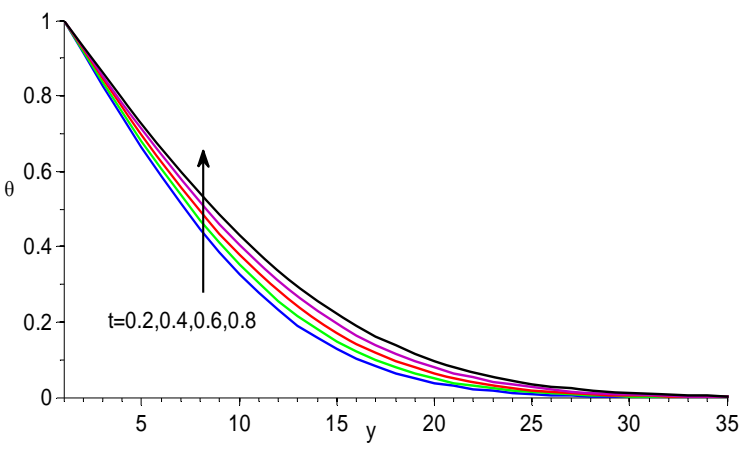

Fig. 14 Effect of time on temperature for $\mathrm{Gr}=5, \mathrm{Gc}=5, \mathrm{M}^{2}=10, \mathrm{Pr}=0.71, \mathrm{Nr}=0.5$.

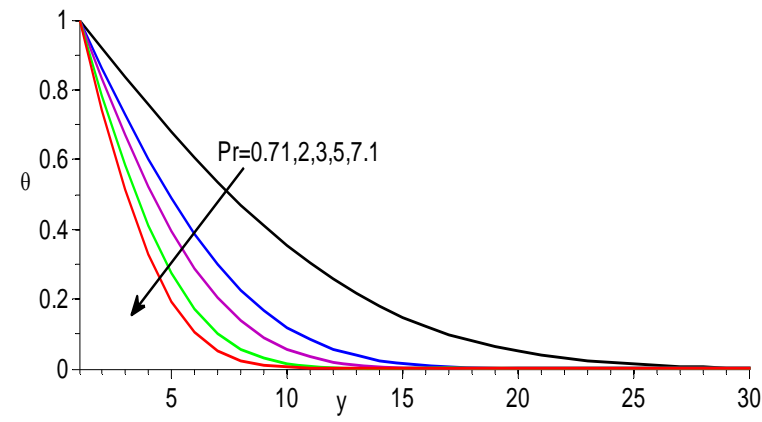

Fig. 15 Effect of Prandtl number on temperature for $\mathrm{Gr}=5, \mathrm{Gc}=5, \mathrm{M}^{2}=10, \mathrm{Nr}=0.5$.

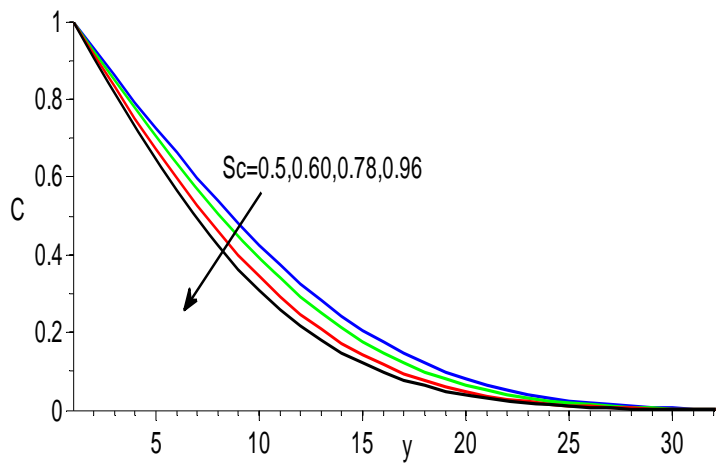

Fig. 16 Effect of Schmidt number on concentration for $\mathrm{Pr}=0.71, \mathrm{Nr}=0.5, \mathrm{So}=1$.

\subsection{Effects of the parameters on concentration profiles:}

Figures 16 and 17 represent the influence of Schmidt number and Soret number on the species concentration. It is seen that the concentration field become thinner under the effect of Schmidt number, but a reverse trend has noticed in the case of Soret effect. The fluid concentration becomes thinner with increasing values of radiation parameter. It is clearly shown in Fig. 18. Fig. 19 reveals the variation of the concentration distribution under the influence of volume fraction parameter. It has observed that the fluid concentration decreases as the solid volume fraction increases. Fig. 20 reveals the variation of the concentration distribution under the influence of time. It has observed that the fluid concentration increases as time increases. 


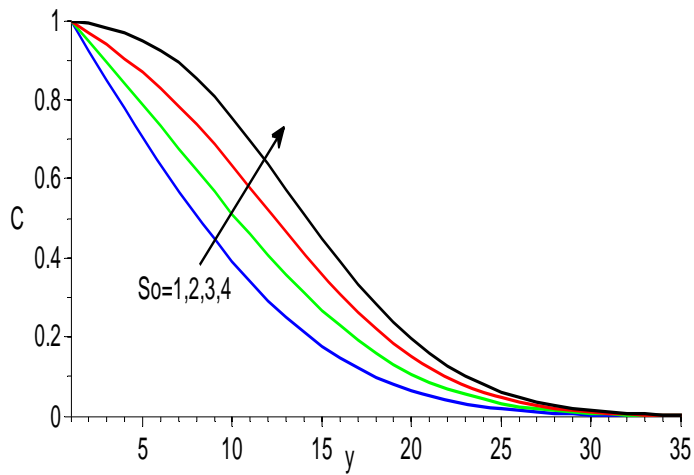

Fig. 17 Effect of Soret number on concentration for $\mathrm{Pr}=0.71, \mathrm{Nr}=0.5, \mathrm{Sc}=0.60$.

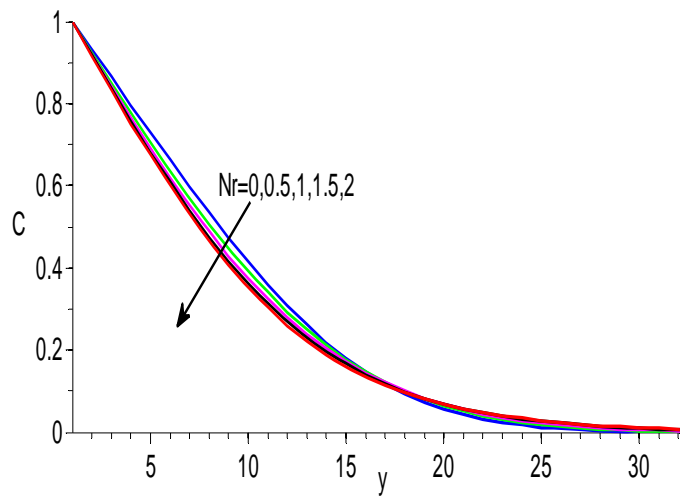

Fig. 18 Effect of radiation parameter on concentration for $\mathrm{Pr}=0.71, \mathrm{Sc}=0.60, \mathrm{So}=1$.

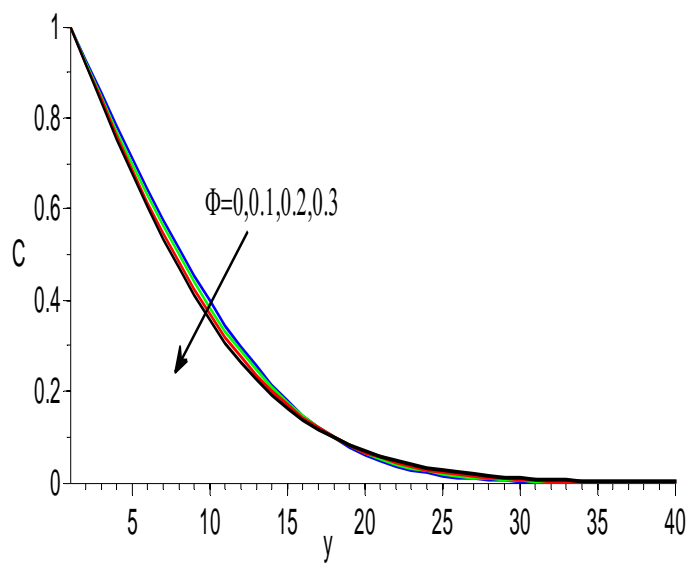

Fig. 19 Effect of Solid volume fraction on concentration for $\mathrm{Pr}=0.71, \mathrm{Nr}=0.5, \mathrm{Sc}=0.60, \mathrm{So}=1$.

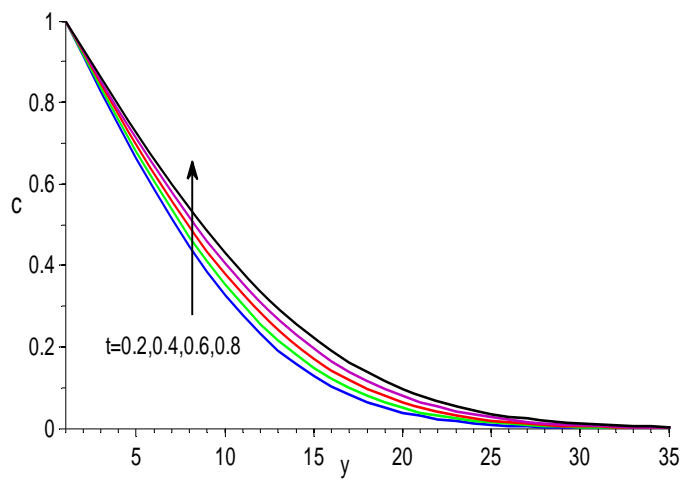

Fig. 20 Effect of time on concentration for $\mathrm{Gr}=5, \mathrm{Gc}=5, \mathrm{M}^{2}=10, \mathrm{Pr}=0.71, \mathrm{Nr}=0.5$.

\subsection{Effects of parameters on shear stress at the plate:}

Researchers are very much interested to evaluate the shear stress (or skin friction) for the sake of engineering purposes. The nondimensional shear stress at the plate $\mathrm{y}=0$ due to the flow is given by the relation

$$
\tau=-\left(\frac{d u}{d y}\right)_{y=0}
$$

Numerical results of the non-dimensional skin friction are presented in Fig. 21-24 under the effects of Grashof number Gr, modified Grashof number Gc, Schmidt number Sc and Soret number. The effects of the Grashof numbers of heat and mass transfer on skin friction are displayed in Fig. 21-22. It can be seen that the skin friction reduces for increasing values of both the numbers for the stationary plate $(\lambda=0)$ and also moving plate $(\lambda= \pm 1)$. This is because the positive buoyancy force acts like a favorable pressure gradient and hence the fluid in the boundary layer gets accelerated. Consequently, the hot fluid near the plate surface passes away faster as Grashof number Gr increases and so the shear stress at the plate reduces. The variation of shear stress with the effect of Schmidt number can be seen in Fig. 23. An increase in Schmidt number leads to an increase in skin friction for the stationary plate $(\lambda=0)$ as well as moving plate $(\lambda= \pm 1)$. Fig. 24 illustrate the behavior of shear stress at the plate which states that the rising values of Soret number results in reduction of shear stress in both the cases of stationary plate $(\lambda=0)$ and moving plate $(\lambda= \pm 1)$.

\subsection{Effects of parameters on the rate of heat transfer at the plate:}

The dimensionless rate of heat transfer in terms of Nusselt number is given by

$$
N u=-\left(\frac{d \theta}{d y}\right)_{y=0}
$$

Numerical values of non-dimensional rate of heat transfer at the plate $\mathrm{y}=0$ are displayed in Figures 25-27 for different values of radiation parameter $\mathrm{Nr}$, Prandtl number $\mathrm{Pr}$ and volume fraction parameter $\phi$. From Fig. 25 it is evident that the rate of heat transfer enhanced with the increasing values of radiation parameter. This happens because as thermal radiation increases, the dominance effect of temperature gradient increases. Consequently, the rate of heat transfer increases. As the Prandtl number increases the rate of heat transfer reduces. It is evident from Fig. 26. Fig. 27 illustrates that the rate of heat transfer increases as volume fraction parameter enlarges. This has been realized with the increase in thermal conductivity under the effect of solid volume fraction of nanoparticles. On the other hand, the thermal boundary layer thickness decreases with increase of nanoparticles volume fraction and in turn the rate of heat transfer increases with increase of solid volume fraction parameter. 


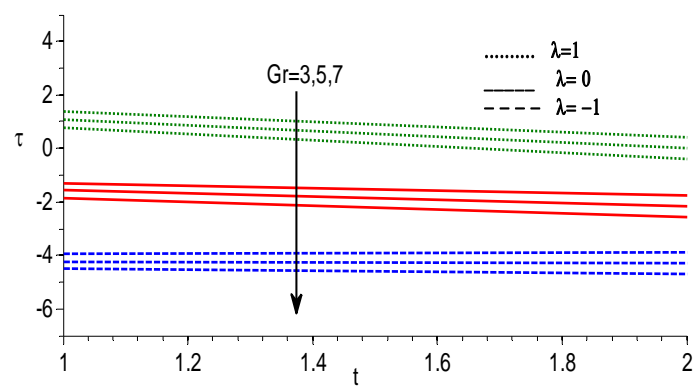

Fig. 21 Effect of Grashof number on skin friction for $\mathrm{Gc}=5, \mathrm{Nr}=0.5, \mathrm{M}^{2}=3, \mathrm{Pr}=0.71, \mathrm{Sc}=0.22, \mathrm{~S}_{0}=0.1$.

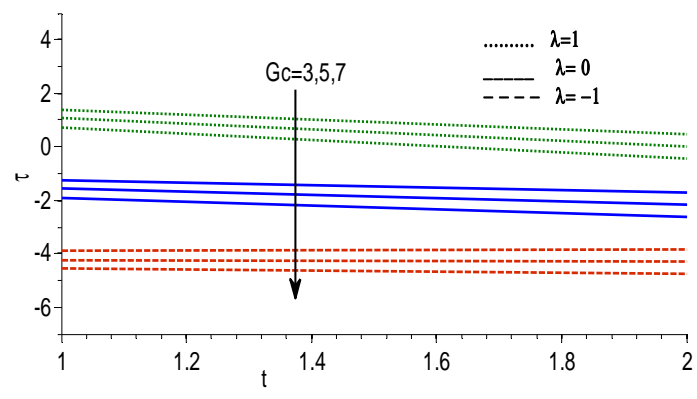

Fig. 22 Effect of modified Grashof number on skin friction for $\mathrm{Gr}=5, \mathrm{Nr}=0.5, \mathrm{M}^{2}=3, \mathrm{Pr}=0.71, \mathrm{Sc}=0.22, \mathrm{~S}_{0}=0.1$.

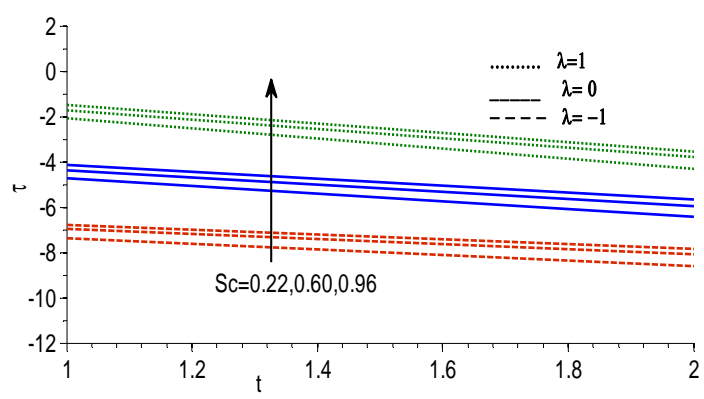

Fig. 23 Effect of Schmidt number on skin friction for $\mathrm{Gr}=15, \mathrm{Gc}=15, \mathrm{Nr}=0.5, \mathrm{M}^{2}=3, \mathrm{Pr}=0.71, \mathrm{~S}_{0}=0.1$.



Fig. 24 Effect of Soret number on skin friction for $\mathrm{Gr}=5, \mathrm{Gc}=5, \mathrm{Nr}=0.5, \mathrm{M}^{2}=3, \mathrm{Pr}=0.71, \mathrm{Sc}=0.96$.
4.6. Effects of parameters on the rate of mass transfer at the plate

The dimensionless rate of mass transfer in terms of Sherwood number is given by

$$
S h=-\left(\frac{d C}{d y}\right)_{y=0}
$$

The effects on the non-dimensional rate of mass transfer at the plate $\mathrm{y}=$ 0 are shown in figures 28-31 for several values of Schmidt number Sc, Soret number So, radiation parameter $\mathrm{Nr}$, and volume fraction parameter $\phi$. Fig. $28 \& 29$ depict the variation of the rate of mass transfer with the effect of Soret number and Schmidt number. The Sherwood number increases with the rising values of Soret number. But a reverse trend is noticed in the case of Schmidt number. Fig. $30 \& 31$ reveal the variation of Sherwood number under the influence of radiation parameter and volume fraction parameter. It is clear that the rate of mass transfer falls down with increasing values of both the parameters.



Fig. 25 Effect of radiation parameter on the Nusselt number for $\mathrm{Gr}=5, \mathrm{Gc}=5, \mathrm{M}^{2}=10, \mathrm{Pr}=0.71$.

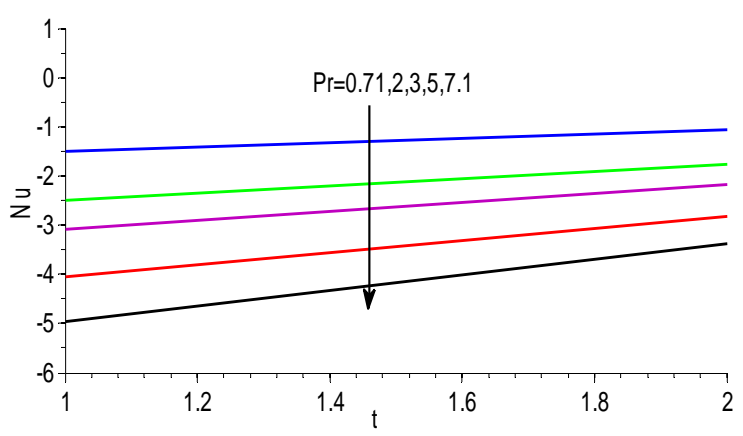

Fig. 26 Effect of Prandtl number on the Nusselt number for $\mathrm{Gr}=5, \mathrm{Gc}=5, \mathrm{M}^{2}=10, \mathrm{Nr}=0.5$.

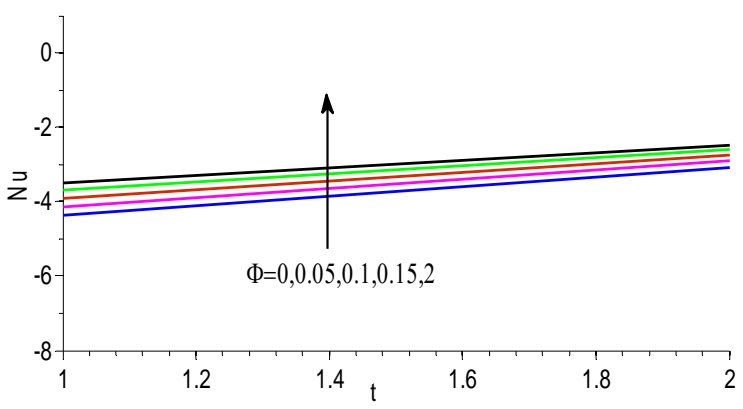

Fig. 27 Effect of solid volume fraction on the Nusselt number for $\mathrm{Gr}=5, \mathrm{Gc}=5, \mathrm{M}^{2}=10, \mathrm{Nr}=0.5, \mathrm{Pr}=0.71$. 


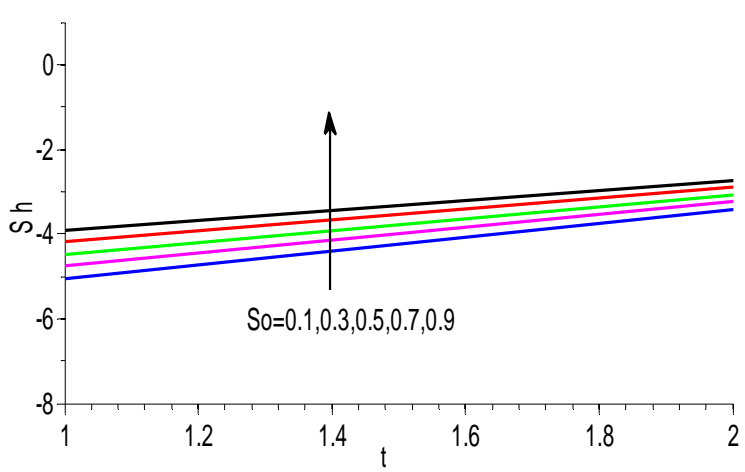

Fig. 28 Effect of Soret number on Sherwood number for $\mathrm{Pr}=0.71, \mathrm{Nr}=0.5, \mathrm{Sc}=0.60$.

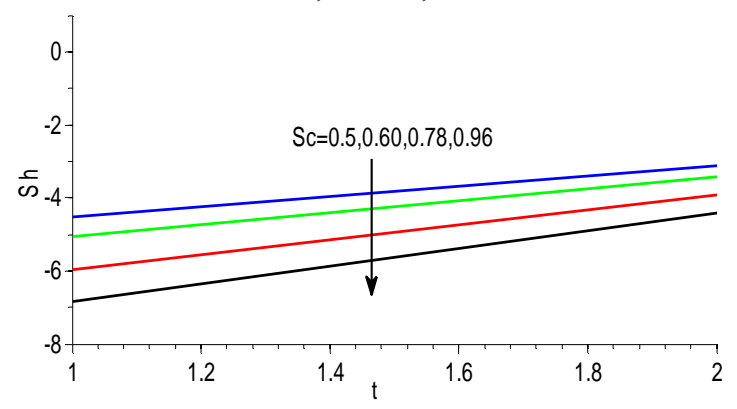

Fig. 29 Effect of Schmidt number on Sherwood number for $\mathrm{Pr}=0.71, \mathrm{Nr}=0.5, \mathrm{So}=0.1$

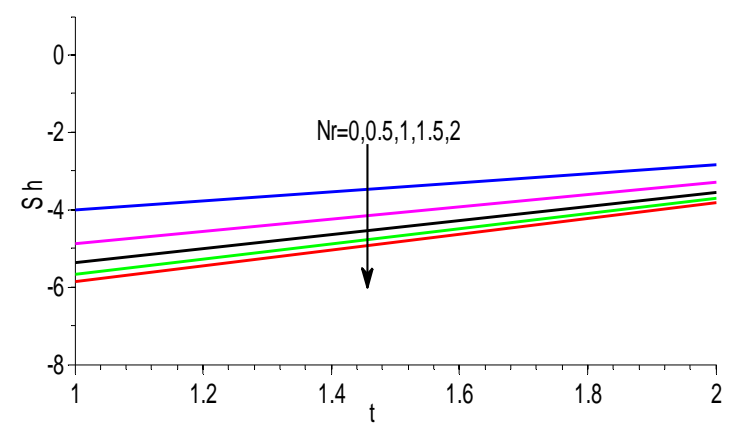

Fig. 30 Effect of radiation parameter on Sherwood number for $\operatorname{Pr}=0.71, \mathrm{Sc}=0.96, \mathrm{So}=1$.

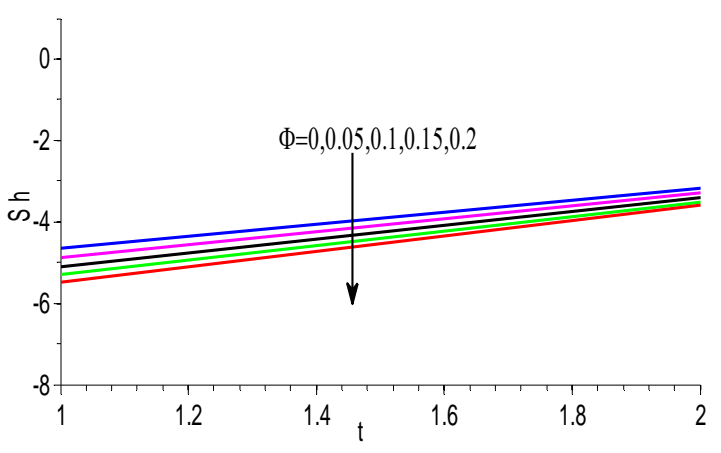

Fig. 31 Effect of Solid volume fraction on Sherwood number for $\mathrm{Pr}=0.71, \mathrm{Nr}=0.5, \mathrm{Sc}=0.96, \mathrm{So}=1$.

\section{CONCLUSIONS}

The purpose of the present work is to analyze the characteristics of magnetohydrodynamic free convective magneto-nanofluid flow through a moving vertical plate in the existence of radiation and thermal diffusion. Rosseland diffusion approximation is considered in the description of radiative heat flux in the energy equation. The expressions for the velocity, temperature and concentration are obtained with the help of finite difference technique. The influences of several parameters encountered in the problem on velocity, temperature and concentration profiles are presented graphically. The effects of the same parameters on the shear stress, rate of heat transfer and rate of mass transfer at the plate are also analyzed graphically. The most interesting points has summarized as follows:

- The fluid velocity increases with increasing radiation parameter and Soret number whereas opposite trend is shown in the case of Prandtl number and Schmidt number.

- An increase in radiation parameter and solid volume fraction leads to enhance the fluid temperature.

- $\quad$ Rising values of Soret number serves to improve the species concentration.

- Increasing values of Schmidt number enhanced the shear stress at the plate but a reverse trend is noticed in the case of Soret number.

- With an increase in radiation parameter and volume fraction parameter the rate of heat transfer enlarged.

- Sherwood number increases for rising values of Soret number and falling values of Schmidt number.

\section{ACKNOWLEDGEMENT:}

The authors are very much thankful to the reviewers for giving valuable suggestions to improve the quality of the manuscript. We also thank the editor for handling this manuscript.

\section{NOMENCLATURE:}

$\mathrm{u}^{*} \quad$ velocity component along the $\mathrm{x}$ - direction $\left[\mathrm{m} \cdot \mathrm{s}^{-1}\right]$

$\mathrm{T}^{*} \quad$ temperature $[\mathrm{K}]$

$\mathrm{C}^{*} \quad$ concentration $[\mathrm{g} / \mathrm{ml}]$

$\mu_{n f} \quad$ dynamic viscosity of the nanofluid [Pa.s]

$\rho_{n f} \quad$ density of the nanofluid [kg.m $\left.{ }^{-3}\right]$

$\sigma_{n f} \quad$ electrical conductivity of the nanofluid $\left[\mathrm{ohm}^{-1} \mathrm{~s}^{-1}\right]$

$k_{n f} \quad$ thermal conductivity of the nanofluid $\left[\mathrm{W} \cdot \mathrm{m}^{-1} \cdot \mathrm{K}^{-1}\right.$ ]

g acceleration due to gravity $\left[\mathrm{m} \cdot \mathrm{s}^{-2}\right]$

$q_{r} \quad$ radiative heat flux

$\left(\rho C_{p}\right)_{n f}$ heat capacitance of the nanofluid

$\beta_{n f} \quad$ thermal expansion coefficient $\left[\mathrm{K}^{-1}\right]$

$\beta_{n f}^{*} \quad$ mass transfer coefficient $[\mathrm{m} / \mathrm{s}]$

$\mathrm{t} \quad$ time [sec]

$\phi \quad$ solid volume fraction of the nanoparticles [m.g]

$\rho_{f} \quad$ density of the base fluid $\left[\mathrm{kg} \cdot \mathrm{m}^{-3}\right]$

$\rho_{s} \quad$ density of the nanoparticles

$\sigma_{f} \quad$ electrical conductivity of the base fluid $\left[\mathrm{ohm}^{-1} \mathrm{~s}^{-1}\right]$

$\sigma_{s} \quad$ electrical conductivity of the nanoparticles

$\mu_{f} \quad$ viscosity of the base fluid[Pa.s]

$\left(\rho C_{p}\right)_{f}$ heat capacitance of the base fluid

$\left(\rho C_{p}\right)_{s}$ heat capacitance of the nanoparticles 
D mass diffusion coefficient

$D_{1} \quad$ thermal diffusion coefficient

$k_{f}$ thermal conductivity of the basefluid $\left[\mathrm{W} \cdot \mathrm{m}^{-1} \cdot \mathrm{K}^{-1}\right.$ ]

$k_{s} \quad$ thermal conductivity of the nanoparticles

Superscript

* Dimensional

\section{REFERENCES:}

Choi, S.U.S., 1995, "Enhancing Thermal Conductivity of Fluids with Nanoparticles Developments and Applications of Non-Newtonian Flows," ASME FED, 231/MD 66, 99-105, 1995.

Eastman, J.A., Choi, S.U.S., Thompson, S.J., Lee, S., Li, S., 1997, "Enhanced Thermal Conductivity through the Development of Nanofluids" in: Komarneni, S., Parker, J.C., Wollenberger, H.J., (Eds.), Nanophase and Nanocomposite Materials II, Materials Research Society, Pittsburgh.

Eastman, J.A., Choi, S. U. S., Li, S., Yu, W., Thompson, L.J., 2001, "Anomalously Increased Effective Thermal Conductivities Of Ethylene Glycol-Based Nanofluids Containing Copper Nanoparticles," App. Phy. Lett., 78, 718-720.

Choi, S.U.S., Zhang, Z.G., Yu, W., Lockwood, F.E., Grulke, E.A., 2011, "Anomalously Thermal Conductivity Enhancement in Nanotube Suspensions," App. Phy. Lett., 79, 2252-2254.

Das, S.K., Putra, N., Thiesen, P., Roetzel, W., 2003, "Temperature Dependence of Thermal Conductivity Enhancement for Nanofluids," Journal of Heat Transfer, 125, 567-574.

http://dx.doi.org/10.1115/1.1571080

Masuda, H., Ebata, A., Teramae, K., Hishinuma, N., 1993, "Alteration of Thermal Conductivity and Viscosity of Liquids by Dispersing Ultrafine Particles," Netsu Busse, 7, 227-233.

Das, S.K., Choi, S.U., 2009, "A Review of Heat Transfer In Nanofluids," Adv. Heat Transfer, 46, 119.

Kakac, S., Pramuanjaroenkij, A., 2009, "Review of Convective Heat Transfer Enhancement with Nanofluids," Int. Journal of Heat Mass Transfer, 52, 3187-3196.

http://dx.doi.org/10.1016/j.ijheatmasstransfer.2009.02.006

Sheikholeslami, M., Ganjia, D.D., Rashidib, M.M., 2014, "Ferrofluid Flow and Heat Transfer in a Semi Annulus Enclosure in the Presence of Magnetic Source Considering Thermal Radiation," Journal of Taiwan Inst. Chem. Eng.

http://dxdoi.org/10.1016/i.jtice.2014.09.026.

Sheikholeslami, M., Hatami, M., Domairry, G., 2014, "Numerical Simulation of Two Phase Unsteady Nanofluid Flow and Heat Transfer Between Parallel Plates in Presence of Time Dependent Magnetic Field," Journal of Taiwan Inst. Chem. Eng.

http://dxdoi.org/10.1016/j.jtice.2014.09.025.

Sheikholeslami, M., Gorji-Bandpya, Vajravelu, K., 2015, "Lattice Boltzmann Simulation of Magnetohydrodynamic Natural Convection Heat Transfer of $\mathrm{Al}_{2} \mathrm{O} 3$-Water Nanofluid in A Horizontal Cylindrical Enclosure with An Inner Triangular Cylinder," Int. Jou. Heat Mass Transfer, 80, 16-25.

http://dx.doi.org/10.1016/j.ijheatmasstransfer.2014.08.090.

Sheikholeslami, M., Abelman, S., Ganji, D.D., 2014, "Numerical Simulation Of MHD Nanofluid Flow And Heat Transfer Considering
Viscous Dissipation," International Journal of Heat Mass Transfer, 79, $212-222$.

http://dx.doi.org/10.1016/j.ijheatmasstransfer.2014.08.004

Kandelousi, M.S., K. K. L., 2014, "Correlation for Simulation of Nanofluid Flow and Heat Transfer in a Permeable Channel," Phys. Lett. A.

http://dx.doi.org/10.1016/j.physleta.2014.09.046

Sheikholeslami, M., and Ganji, D.D., 2014, "Ferrohydrodynamic and Magnetohydrodynamic Effects on Ferrofluid Flow and Convective Heat Transfer," Energy. 75, 400-410.

http://dx.doi.org/10.1016/j.energy.2014.07.089

Sheikholeslami, M., and Gorji-Bandpya, M., 2014, "Free Convection of Ferro Fluid in a Cavity Heated from Below in the Presence of an External Magnetic Field," Powder Technol. 256, 490-498.

http://dx.doi.org/10.1016/j.powtec.2014.01.079

Hamad, M.A.A., Pop, I., 2011, "Unsteady MHD Free Convection Flow Past a Vertical Permeable Flat Plate in a Rotating Frame of Reference with Constant Heat Source in a Nanofluid," Heat Mass Transfer. 47, $1517-1524$

http://dx.doi.org/10.1007/s00231-011-0816-6

Hamad, M.A.A., Pop, I., Ismail, I.A.M., 2010, "Magnetic Field Effects on Free Convection Flow of a Nanofluid Past a Vertical Semi Infinite Flat Plate," Nonlinear Anal. Real World Appl. 12 (3), 1338-1346. http://dx.doi.org/10.1016/j.nonrwa.2010.09.014.

Hayat, T., Mustafa, M., and Pop, I., 2010, "Heat And Mass Transfer For Soret And Dufour Effects On Mixed Convection Boundary Layer Flow Over A Stretching Vertical Surface In A Porous Medium Filled With A Viscoelastic Fluid," Comm. Nonlinear Sci. Num. Sim. 15, 1183 1196.

http://dx.doi.org/10.1016/j.cnsns.2009.05.062.

Chamkha, A.J., and Aly, A.M., 2011, "MHD Free Convection Flow of a Nanofluid Past a Vertical Plate in the Presence of Heat Generation or Absorption Effects," Chem. Eng. Comm.198, 425- 441.

http://dx.doi.org/10.101080/00986445.2010.520232

Turkyilmazoglu, M., 2012, "Exact Analytical Solutions for Heat And Mass Transfer of MHD Slip Flow in Nanofluids," Chem. Eng. Sci. 84, 182-187.

http://dx.doi.org/10.1016/j.ces.2012.08.029

Vajravelu, K., Prasad, K.V., and Chiu-On Ng, 2013, "Unsteady Convective Boundary Layer Flow of a Viscous Fluid at a Vertical Surface with Variable Fluid Properties," Non-linear Analysis: Real WorldApplications 14, 455-464.

http://dx.doi.org/10.1016/j.nonrwa.2012.07.008

Prakash, J., Bhanumathi, D., Vijaya Kumar, A. G., and Varma, S. V. K., 2013, "Diffusion-Thermo and Radiation Effects on Unsteady MHD Flow through Porous Medium Past an Impulsively Started Infinite Vertical Plate with Variable Temperature and Mass Diffusion" Trans. Porous. Med., 96, 135-151.

http://dx.doi.org/10.1007/S11242-012-0078-x

Seth, G. S., \& Ansari, Md. S., 2010, "MHD Natural Convection Flow Past An Impulsively Moving Vertical Plate With Ramped Wall Temperature In The Presence Of Thermal Diffusion With Heat Absorption,” Int. J. Appl. Mech. Eng., 15(1), 199-215.

Khan, W.A., Makinde, O.D., and Khan, Z.H., 2014, "MHD Boundary Layer Flow Of A Nanofluid Containing Gyro Tactic Micro Organisms 
Past A Vertical Plate With Navier Slip," International journal of heat and mass transfer, 74, 285-291.

http://dx.doi.org/10.1016/j.ijheatmasstransfer.2014.03.026

Srinivasacharya, D. and Kaladhar, K., 2012a, "Mixed Convection Flow of Couple Stress Fluid between Parallel Vertical Plates with Hall and Ion-Slip Effects," Commun Nonlinear Sci Numer Simulat, 7(6), 24472462.

http://dx.doi.org/10.1016/j.cnsns.2011.10.006.

Kaladhar, K., Srinivasacharya, D., 2014 "Effects of Thermal and Solutal Stratification on Mixed Convection Flow along a Vertical Plate Saturated With Couple Stress Fluid," Frontiers in Heat and Mass Transfer (FHMT), 5, 11.

http://dx.doi.org/10.5098/hmt.5.11.

Srinivas, J. and Ramana Murthy, J.V., 2015, “Thermodynamic Analysis For The MHD Flow of Two Immiscible Micropolar Fluids between Two Parallel Plates," Frontiers in Heat and Mass Transfer (FHMT), 6, 4.

http://dx.doi.org/10.5098/hmt.6.4.

Mahian, O., Mahmud, S., and Pop, I., 2012, "Analysis of First, Second Laws of Thermodynamics between Two Isothermal Cylinders with Relative Rotation in the Presence of MHD Flow," Int. Journal of Heat Mass Transfer, 55(17-18), 4808-4816.

http://dx.doi.org/10.1016/j.ijheatmasstransfer.2012.04.048

Yu, S.H., Jang, D., and Lee, K.S., 2012, "Effect Of Radiation in a Radial Heat Sink under Natural Convection," International Journal of Heat and Mass Transfer, 55, 505-509.

http://dx.doi.org/10.1016/j.ijheatmasstransfer.2011.09.026.

Umamaheswar, M., Raju, M.C., Varma, S.V.K., 2015 "Analysis of MHD Transient Free Convection Flow of a Newtonian Fluid Past an Infinite Vertical Porous Plate," Frontiers in Heat and Mass Transfer, 618, 1-7.

http://dx.doi.org/10.5098/hmt.6.18.

Das, S., Jana, R.N., 2015, "Natural Convective Magneto-Nanofluid Flow and Radiative Heat Transfer Past a Moving Vertical Plate," Alexandria Engg. Journal., 54, 55-64. http://dx.doi.org/10.1016/j.aej.2015.01.001

Ferdows, M., Khan, M.S., Alam, M.M., and Sun, S., 2012, "MHD Mixed Convective Boundary Layer Flow of A Nanofluid through A Porous Medium due to an Exponentially Stretching Sheet," Mathematical problems in Engineering, 3(7), 2551-155.

http://dx.doi.org/10.1155/2012/408528
Ferdows, M., Khan, M.S., Alam, M.M., Bég, O.A., Alam, M.M., 2014, "Numerical Study of Transient Magnetohydrodynamic Radiative Free Convection Nanofluid Flow from a Stretching Permeable Surface," Journal of Process Mechanical Engineering, 228(3), 181-196.

http://dx.doi.org/10.1177/0954408913493406

Khan, M.S., Karim, I., Islam, M.S. and Wahiduzzaman, M., 2014, "MHD Boundary Layer Radiative, Heat Generating and Chemical Reacting Flow past a Wedge Moving in a Nanofluid," Nano Convergence, 1:20, 1-13.

http://www.nanoconvergencejournal.com/content/1/1/20

Khan, M.S., Karim, I., Islam, M.S., Wahiduzzaman, M., and Alam, M.M., 2014, "Heat Generation Effects on Unsteady Mixed Convection Flow from a Vertical Porous Plate with Induced Magnetic Field," Procedia Engineering, 90, 238-244.

http://dx.doi.org/10.1016/j.proeng.2014.11.843

Wahiduzzaman, M., Khan, M.S., Karim, I., Biswas, P., and Uddin, M.S., 2015 "Viscous Dissipation and Radiation effects on MHD Boundary Layer Flow of a Nanofluid past a Rotating Stretching Sheet". Applied Mathematics, 6, 547-567.

http://dx.doi.org/10.4236/am.2015.63050

Wahiduzzaman, M., Khan, M.S., Karim, I., 2015, "MHD Convective Stagnation Flow Of Nanofluid Over A Shrinking Surface with Thermal Radiation, Heat Generation and Chemical Reaction," Procedia Engineering, 105, 398-405.

http://dx.doi.org/10.1016/j.proeng.2015.05.025

Khan, M.S., Karim, I., Ali, L.E. and Islam, A., 2012, "MHD Free Convection Boundary layer Unsteady Flow of a Nanofluid along a stretching sheet with thermal Radiation and Viscous Dissipation Effects," International Nano Letters, 2:24, 1-9.

http://www.inl-journal.com/content/2/1/24

Beg, O.A., Khan, M.S., Karim, I., Alam, M.M., Ferdows, M., 2014, "Explicit Numerical Study of Unsteady Hydromagnetic Mixed Convective Nanofluid Flow from an Exponential Stretching Sheet in Porous Media," Applied Nanoscience, 4, 943-957.

http://dx.doi.org/10.1007/s13204-013-0275-0

Reddy, S.H., Raju, M.C., Keshava Reddy, E., 2016, "Radiation Absorption and Chemical Reaction Effects on MHD Flow of Heat Generating Casson Fluid Past Oscillating Vertical Porous Plate," Frontiers in Heat and Mass Transfer, 7, 21. http://dx.doi.org/10.5098/hmt.7.21 